\title{
Application des méthodes d'analyses statistiques multivariées à l'étude de la minéralisation des eaux de la zone de Zinder (Sud-Est du Niger)
}

\author{
Haoua AMADOU*, Mahaman Sani LAOUALI et Abdou Salam MANZOLA \\ Laboratoire de Chimie de l'eau, Faculté des Sciences et Techniques, \\ Université Abdou Moumouni de Niamey, BP 10662 Niamey, Niger. \\ "Auteur correspondant ; E-mail: haoua_amadou2000@yahoo.fr; Tel : (+227) 96547254.
}

\section{RESUME}

Les eaux souterraines constituent l'une des principales sources d'approvisionnement en eau potable de la population de la région de Zinder. La présente étude s'intéresse à la qualité des eaux de trois aquifères (le continental Hamadien, le Granite Altéré et les Sables du Manga) de la région de Zinder. L'objectif est d'améliorer les connaissances sur les nappes souterraines plus particulièrement déterminer l'origine de la minéralisation de ces eaux. Une série d'analyses physico-chimiques et microbiologiques effectuées sur des échantillons prélevés dans les puits et les forages a été réalisée. Les résultats de ces analyses ont été traités à partir d'une méthode hydro-chimique qui utilise le diagramme triangulaire de Piper et des méthodes statistiques multi variées dont l'Analyse en Composantes Principales Normées (ACPN) et la Classification Hiérarchique Ascendante (CHA). Les résultats de cette étude montrent que la température des eaux de la région varie entre $28,2{ }^{\circ} \mathrm{C}$ et $30,5^{\circ} \mathrm{C}$, avec une moyenne de $29,45 \pm 0,1{ }^{\circ} \mathrm{C}$. Les eaux ont un pH qui varie entre 6,5 et 7,48 unités $\mathrm{pH}$, avec une moyenne de $6,90 \pm 0,2$. Celles-ci sont douces, avec une dureté moyenne de 8,08 ${ }^{\circ} \mathrm{F}$. Les eaux sont riches en fer et ont des teneurs qui varient entre $0,026 \mathrm{mg} / \mathrm{l}$ et $2,14 \mathrm{mg} / \mathrm{l}$ soit une moyenne de $0,36 \mathrm{mg} / \mathrm{l}$. Les eaux de la zone d'étude se répartissent en quatre grandes familles. Il s'agit des eaux bicarbonatées calciques et magnésiennes qui sont les plus importantes (50\%), les eaux bicarbonatés sodi-potassiques (30\%), les eaux chlorurées calciques et magnésiennes (10\%) et les eaux sulfatées sodi-potassiques (10\%). L'Analyse en Composantes Principales et la Classification Ascendante Hiérarchique indiquent que la minéralisation des eaux étudiées est contrôlée par deux grands phénomènes : la minéralisation temps de séjour qui se traduit par l'hydrolyse des minéraux et le phénomène d'oxydo-réduction. Cette étude montre également que les ressources en eaux étudiées, principalement les eaux des puits sont fortement influencées par les activités anthropiques, avec la présence des coliformes fécaux de type Escherichia coli et de streptocoques fécaux qui sont la conséquence d'une pollution d'origine humaine récente.

(C) 2014 International Formulae Group. All rights reserved.

Mots clés: Analyse en Composantes Principales Normées, Classification Hiérarchique Ascendante, minéralisation, eaux souterraines, Zinder.

\section{INTRODUCTION}

L'utilisation intensive des ressources naturelles et l'accroissement des activités humaines ont engendré de graves problèmes sur la qualité des eaux souterraines (Mor et al., 2006). En effet, les eaux souterraines sont de plus en plus utilisées par les populations en général et particulièrement par le monde rural 
pour leurs besoins domestiques. Mais, très souvent ces eaux sont d'une coloration rougeâtre et ont un goût désagréable, d'où la nécessité d'une bonne connaissance de la qualité de ces eaux.

La composition chimique d'une eau issue du milieu naturel est très variable. Elle dépend, entre autres, de la nature géologique du sol d'où elle provient et des substances réactives qu'elle pourrait rencontrer lors de l'écoulement (Jain, 2005; Kortatsi, 2007). Toutefois, la qualité des eaux peut être altérée lorsque des substances extérieures entrent en contact avec la nappe aquifère. La compréhension des propriétés hydrauliques des couches aquifères et la caractérisation hydro-chimique de l'eau qu'elles contiennent sont nécessaires pour la planification et la gestion des ressources en eau souterraine.

La présente étude s'intéresse à la qualité des eaux de trois aquifères (le continental Hamadien, les eaux du granite altéré et les eaux du sable de Manga) de la région de Zinder. L'objectif de cette étude est de caractériser le contexte hydrogéologique de ces aquifères, pour une compréhension de l'acquisition de la minéralisation des eaux souterraines de cette région. La méthodologie appliquée est basée sur la détermination de la Classification hydrogéochimique des eaux souterraines à partir du diagramme de Piper et l'utilisation des méthodes statistiques multi variées (Analyse en Composantes Principales Normées (ACPN) et Classification Hiérarchique Ascendante (CHA)).

\section{MATERIEL ET METHODES}

\section{Présentation de la zone d'étude}

Située dans la partie Sud-Est du territoire nigérien, la région de Zinder s'étend en latitude entre $12^{\circ} 48^{\prime}$ et $17^{\circ} 30^{\prime}$ Nord et en longitude entre $7^{\circ} 20^{\prime}$ et $12^{\circ} 00^{\prime}$ Est (Figure 1).

Le climat de la région est de type sahélien dans le Sud avec une transition vers un climat de type désertique dans le Nord. Il est caractérisé par deux saisons distinctes: une saison des pluies de quatre à cinq mois de mijuin à septembre ou octobre, avec des températures moyennes de 30 à $35^{\circ} \mathrm{C}$ et une longue saison sèche avec des températures variant entre $20^{\circ} \mathrm{C}$ en décembre et plus de 40 ${ }^{\circ} \mathrm{C}$ en mai. Les variations spatiales de la pluviométrie sont importantes de même que les variations annuelles.

La région de Zinder constitue un seuil où le cristallin affleure en boutonnière (Greigert et Pougnet, 1967 ; Ousmane, 1978) entre les bassins des Iullemmenden à l'Ouest et du Tchad à l'Est. Il faut distinguer trois grands ensembles géologiques: le socle cristallin, le continental hamadien, et les sables quaternaires.

Le continental hamadien : il est formé par un ensemble de dépôts fluviatiles d'âge crétacé supérieur constitués de grès fins à grossiers. Ces grès à ciment argileux de couleur blanche, brune et violette présentent des stratifications entrecroisées et de fréquentes variations latérales de faciès. Le continental hamadien forme le massif du koutous au Nord du Damagaram-Mounio et les buttes qui en constituent le prolongement à l'Ouest et au Sud.

Les sables quaternaires: les dépôts sableux recouvrent de façon irrégulière toute la région ne laissant affleurer qu'assez rarement le socle. Parfois, ils ne constituent qu'un nappage de quelques centimètres à quelques décimètres d'épaisseur ou bien ils sont agencés en dunes importantes. Celles-ci se localisent surtout au Nord, à l'Est et au Sud de la région. Les principales ressources en eaux souterraines sont exploitées par des puits et forages.

\section{Méthodologie}

Les analyses physico-chimiques ont été réalisées sur les eaux de forages et puits captant les nappes du Continental Hamadien, 
Granite Altéré et du Sables du Manga. Les prélèvements sont réalisés et conditionnés dans des flacons spécialement préparés à cet effet. Les prélèvements pour les forages munis de robinets sont effectués après avoir fait couler l'eau dans le vide pendant environ trois minutes. Les flacons d'échantillonnage sont ensuite remplis à des fins d'analyse. Pour les puits, les prélèvements sont effectués à l'aide d'un échantillonneur spécialement conçu. Le dispositif d'échantillonnage est soigneusement lavé à l'eau distillée avant chaque prélèvement. La quasi-totalité des points d'eau des aquifères est destinée à l'alimentation en eau potable.

Les paramètres physiques à savoir le $\mathrm{pH}$, la température, la conductivité, la turbidité et la couleur, ont été mesurés in situ respectivement à l'aide d'un $\mathrm{pH}$-mètre, un colorimètre portatif qui couvre les longueurs d'onde de 400 à 800 nanomètres équipé d'une lampe à filament de tungstène, un conductimètre de marque WTW 330 et d'un turbidimètre portatif de marque Hach 2100 Q. La couleur a été déterminée en utilisant la Norme Française NF $\mathrm{T}$ 90-034. Les paramètres chimiques : $\mathrm{Fe}^{2+}$ et $\mathrm{Na}^{+} ; \mathrm{Cl}^{-}, \mathrm{SO}_{4}{ }^{2-}$ ; $\mathrm{F}^{-} ; \mathrm{HCO}_{3}^{-} ; \mathrm{NO}_{3}^{-}$et $\mathrm{NO}_{2}^{-}$ont été analysés dans le Chef-lieu de la région où un laboratoire a été spécialement aménagé. Ces analyses ont été réalisées à l'aide d'un spectrophotomètre en utilisant les méthodes classiques préconisées par les normes françaises AFNOR. En ce qui concerne la microbiologie, l'échantillonnage a été réalisé dans des tubes à essai stérilisés par autoclavage à $121{ }^{\circ} \mathrm{C}$ pendant 20 minutes. Les tests bactériologiques ont été ceux des coliformes fécaux et des streptocoques fécaux, qui sont des tests recommandés par l'OMS pour le cas des eaux de boisson.

La méthode utilisée pour isoler et déterminer ces bactéries, a été la technique par filtration. C'est une méthode très sensible qui permet de déterminer les bactéries à des faibles concentrations. Elle a consisté à filtrer un volume de $10 \mathrm{ml}$ d'échantillon sur une membrane en cellulose de $45 \mathrm{~cm}$ de diamètre et de taille de pores $0,45 \mu \mathrm{m}$. Pour faciliter le comptage des colonies, des filtres stériles et quadrillés ont été utilisés. Une rampe de filtration à trois postes en inox toujours stérilisée par flambage à l'alcool avant toute filtration a permis de filtrer les échantillons. Les filtres ont été ensuite déposés sur un milieu nutritif contenu dans des boîtes de pétri. Ces boîtes de pétri ont été ensuite placées dans des incubateurs réglés à $37{ }^{\circ} \mathrm{C}$ pour les streptocoques et à $44{ }^{\circ} \mathrm{C}$ pour les coliformes. La lecture a été faite après 24 heures et 48 heures de séjour dans les incubateurs.

Aussitôt prélevés, tous les échantillons ont été placés dans une glacière contenant de la glace et directement acheminés sur les lieux d'analyse au plus tard quatre (4) heures de temps après les prélèvements. Les échantillons d'eau destinés aux analyses chimiques ont été prélevés dans 10 cantons de la ville de Zinder (Figure1). Le choix des sites a été basé sur la construction récente de puits ou de forages et sur les points d'eau les plus utilisés par la population. Les aquifères échantillonnés ont été répartis comme suit :

- Le continental Hamadien avec trois

(3) forages à Dan-Goudao, Koundoumawa, Dakouma-idiari, et un puits (1) Kaotchouloum ;

- Le Granite Altéré: 3 forages échantillonnés dans les différentes localités à savoir Birnin-Kazoe, Toumnia, DogoMaikassoua ;

- Les Sables du Manga: 3 forages (Male-Male, Zagari, Soubdou) ont été échantillonnés.

\section{Analyse statistique}

L'ensemble des données collectées sur les eaux souterraines de la région a fait l'objet d'une analyse hydro-chimique et d'une analyse statistique.

L'analyse hydro-chimique a été réalisée à l'aide du digramme de Piper pour caractériser les faciès géochimiques des eaux prélevées. $\mathrm{Ce}$ diagramme est très fréquemment utilisé et donne de très bons résultats (Oga et al., 2009 ; Kouassi et al., 
2010 ; Yao et al., 2010 ; Ahoussi et al., 2011 ; Ahoussi et al., 2012; Ahoussi et al., 2013 ; Eblin et al., 2014). Les différents traitements ont été possibles grâce au logiciel DIAGRAMME ${ }^{\mathrm{TM}}$.

L'approche de la statistique multi variée a été réalisée à partir d'une Analyse en Composantes Principales Normées (ACPN) et d'une Classification Hiérarchique Ascendante (CHA). L'analyse statistique a été réalisée sur 10 échantillons et 12 variables (la conductivité électrique (C.E), le pH, la dureté, la turbidité,
$\mathrm{Na}^{+}, \mathrm{F}^{-}, \mathrm{Fe}^{2+}, \mathrm{Cl}^{-}, \mathrm{HCO}_{3}{ }^{-}, \mathrm{SO}_{4}{ }^{2-}, \mathrm{NO}_{3}{ }^{-}$et $\mathrm{NO}_{2}{ }^{-}$) à l'aide du logiciel XLSTAT 2014. Cette analyse permet de synthétiser et de classer un nombre important de données afin d'en extraire les principaux facteurs qui sont à l'origine de l'évolution simultanée des variables et de leur relation réciproque (Biémi, 1992). Elle permet de mettre en évidence les ressemblances entre deux ou plusieurs variables chimiques au cours de leur évolution.

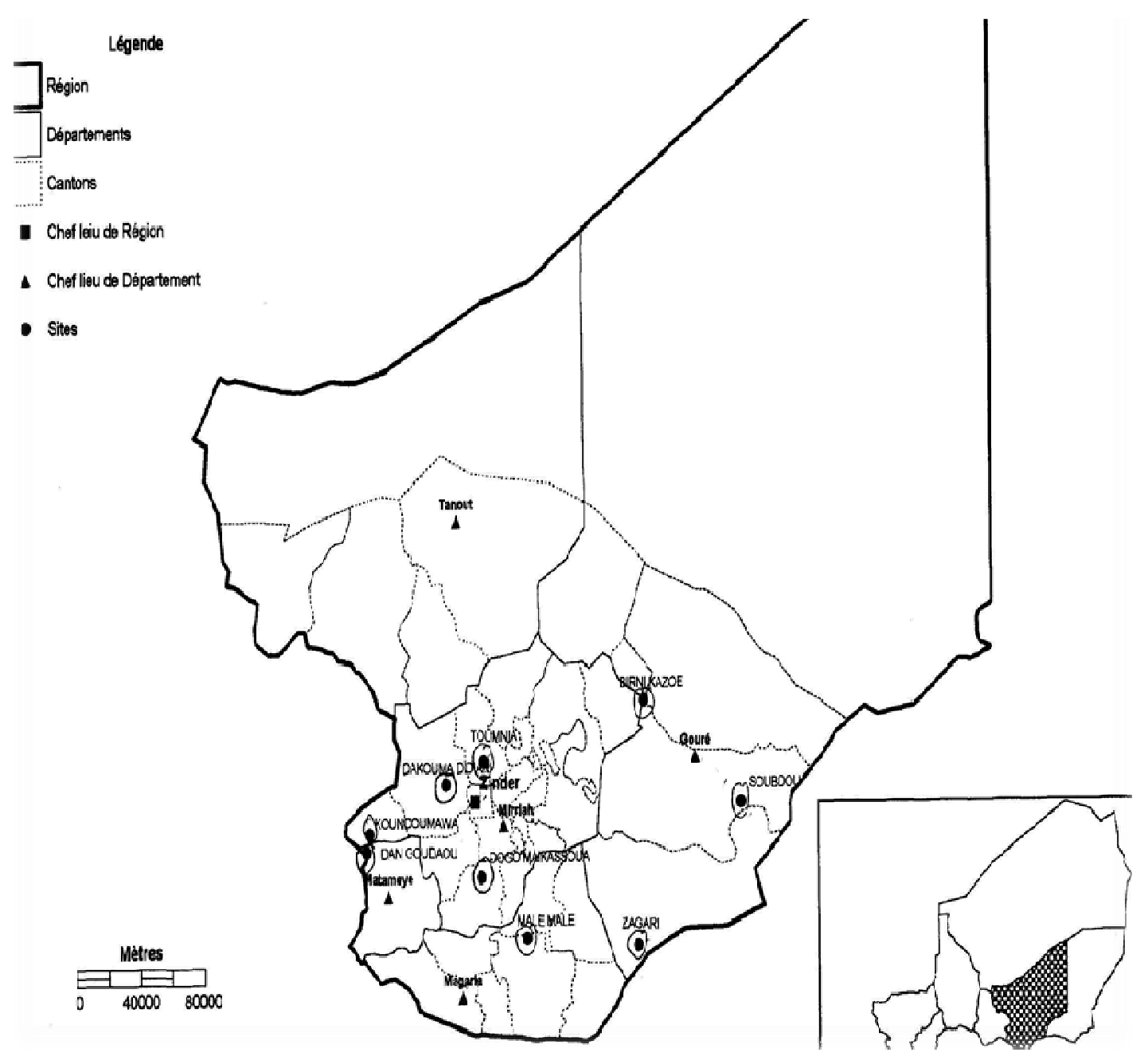

Figure 1 : Localisation des points de prélèvement d'eau souterraine. 


\section{RESULTATS}

\section{Résultats des analyses physico-chimiques}

Les résultats des analyses physicochimiques réalisées sur les eaux souterraines de la région de Zinder (Tableau 1) montrent que la température des eaux souterraines varie entre $28,2{ }^{\circ} \mathrm{C}$ et $30,5^{\circ} \mathrm{C}$, avec une moyenne de $29,45 \pm 0,1^{\circ} \mathrm{C}$. Le $\mathrm{pH}$ des eaux représente l'intensité de l'acidité ou de l'alcalinité et mesure la concentration en ions hydronium dans l'eau. L'intervalle de valeurs de $\mathrm{pH}$ recommandé par l'O.M.S est 6,5-8,5. Le pH des eaux analysées varie entre 6,5 et 7,48 unités $\mathrm{pH}$, avec une moyenne de $6,90 \pm 0,2$. $\mathrm{La}$ conductivité électrique des eaux souterraines de la région varie entre 89 et $1955 \mu \mathrm{S} . \mathrm{cm}^{-1}$, avec une valeur moyenne de $463,9 \pm 37 \mu \mathrm{S} . \mathrm{cm}^{-1}$. Les eaux à forte conductivité sont celles des localités de Birnin-Kazoe (1955 $\mu$ S. $\left.\mathrm{cm}^{-1}\right)$, Soubdou (724 $\left.\mu \mathrm{S} . \mathrm{cm}^{-1}\right)$ et Male-Male $\left(517 \mu \mathrm{S} . \mathrm{cm}^{-1}\right)$. La dureté des eaux souterraines de la région varie entre $2,5^{\circ} \mathrm{F}$ et $18^{\circ} \mathrm{F}$, soit une moyenne de $8,08 \pm 0,9^{\circ} \mathrm{F}$. Cela indique que les eaux de la région sont dans l'ensemble douces à l'exception de celles des localités de BirninKazoe $\left(18^{\circ} \mathrm{F}\right)$ et de Male-Male $\left(15,5^{\circ} \mathrm{F}\right)$. Le fer est indispensable pour le corps humain mais de très fortes concentrations affectent les propriétés organoleptiques de l'eau et tâchent aussi le linge. La valeur guide O.M.S du fer total dans l'eau de boisson est $0,3 \mathrm{mg} / \mathrm{l}$. les résultats des analyses montrent que la concentration des ions $\mathrm{Fe}^{2+}$ varie entre 0,026 $\mathrm{mg} / \mathrm{l}$ et $2,14 \mathrm{mg} / \mathrm{l}$ soit une moyenne de 0,36 $\mathrm{mg} / \mathrm{l}$.

\section{Diagramme de Piper}

La représentation des résultats d'analyses chimiques dans le diagramme de Piper a permis d'identifier quatre familles d'eau (Figure 2). La famille des eaux bicarbonatées calciques et magnésiennes sont les plus importantes et représentent $50 \%$ des eaux étudiées. Elles se rencontrent principalement au niveau des eaux des forages des localités telles que Dan-Goudao,
Dakouma-Didiari, Toumnia, Dogo Maikassoua et du puits de Kaotchouloum. La famille des eaux bicarbonatées sodipotassiques (30\%) s'observent dans les eaux des localités de Koundoumawa, Birnin-Kazoe, Zagari. Celle des eaux chlorurées calciques et magnésiennes (10\%) de la localité de MaleMale et celle des eaux sulfatées sodipotassiques $(10 \%)$ observées à Soubdou. Les eaux de la région sont donc caractérisées par une prédominance des ions bicarbonates sur les ions chlorures et les ions sulfates qui se retrouvent dans les eaux avec des teneurs inférieures à celles de l'OMS (250mg/l).

\section{Résultats de l'Analyse en Composantes Principales Normées (ACPN)}

Les résultats de l'Analyse en Composantes Principales Normées sont présentés dans les Tableaux 2, 3 et Figure 3. La liaison existant entre toutes les variables prises deux à deux et les coefficients de corrélation entre ces différentes variables sont donnés par la matrice de corrélation (Tableau 2). Le Tableau 2 montre que la conductivité est fortement corrélée avec les éléments $\mathrm{Na}^{+}(0,98), \mathrm{HCO}_{3}^{-}(0,96), \mathrm{Cl}^{-}(0,79)$, dureté $(0,81), \mathrm{NO}_{3}^{-}(0,74)$ et corrélé à un degré moindre avec $\mathrm{SO}_{4}{ }^{2-}(0,65)$. Le coefficient de corrélation entre le $\mathrm{pH}$ et le $\mathrm{HCO}_{3}{ }^{-}$est de 0,70 . La couleur présente de fortes corrélations avec la turbidité $(0,99)$ et le $\mathrm{Fe}^{2+}(0,93)$. Les ions $\mathrm{SO}_{4}{ }^{2-}$ présentent une corrélation avec le $\mathrm{HCO}_{3}^{-}(0,7)$ et sont corrélés à un degré moindre avec le $\mathrm{Na}^{+}(0,61)$. Les ions $\mathrm{HCO}_{3}{ }^{-}$ sont fortement corrélés avec le $\mathrm{Na}^{+}(0,96)$, la dureté $(0,73)$ et sont corrélés à un degré moindre avec le $\mathrm{Cl}^{-}(0,66)$. Les ions $\mathrm{Cl}^{-}$sont corrélés avec $\mathrm{Na}^{+}(0,72)$ et la dureté $(0,87)$. Enfin, la dureté est corrélée avec le $\mathrm{Na}^{+}$ $(0,71)$. Ces fortes corrélations entre ces variables mettent en évidence la similarité des phénomènes à l'origine de la mise en circulation de ces ions dans les eaux souterraines de la zone de Zinder.

L'analyse des résultats de l'ACPN de l'espace des variables du plan factoriel F1-F2 
(Figure 3) montre que ce plan factoriel F1-F2 exprime $67,72 \%$ de la variance exprimée. Le Tableau 3 présente les valeurs propres, les variances exprimées pour chaque facteur et leur cumul. Le facteur F1, avec une variance exprimée de $45,38 \%$, est le plus important des facteurs, et le facteur F2 exprime $22,34 \%$ de la variance.

Le facteur $\mathrm{F} 1$ est déterminé par $\mathrm{CE}$, $\mathrm{pH}, \mathrm{HCO}_{3}^{-}, \mathrm{SO}_{4}{ }^{2-}, \mathrm{Cl}^{-}, \mathrm{NO}_{3}{ }^{-}$et la dureté (rattachée principalement à la quantité de calcium et de magnésium dans l'eau). Les éléments qui définissent ce facteur proviennent d'une longue durée de mise en solution suite au contact «eau-roche». Ces éléments qui présentent des variations continues sur de longues périodes caractérisent la minéralisation de l'eau. La forte corrélation qui existe entre ces différentes variables et leur proximité dans le cercle de communauté montrent que la mise en solution de ces différents ions est régie par le même phénomène qui est l'hydrolyse. En effet, l'hydrolyse étant un processus lent, le facteur F1 rend compte des conditions d'acquisition du chimisme de l'eau. Le facteur F1 exprime donc le phénomène de minéralisation-temps de séjour. Le regroupement de la majorité des variables, autour de cet axe montre l'influence de l'altération-hydrolyse dans la mise en solution des ions.

Le facteur 2 est représentatif du fer $\left(\mathrm{Fe}^{2+}\right)$, de la couleur et de la turbidité. Ce qui montre que le facteur 2 est lié au phénomène d'oxydo-réduction.

La représentation graphique dans l'espace factoriel des unités statistiques (Figure 3) rend compte de la répartition des points d'eau en fonction des différents facteurs F1-F2. L'analyse de ce graphe montre deux principaux regroupements des points d'eau :

La classe 1 regroupe les échantillons d'eaux, ayant les plus fortes conductivités électriques dont l'acquisition ionique est sous le contrôle de la minéralisation-temps de séjour. Cette classe concerne $30 \%$ des eaux. Ces eaux sont riches en bicarbonates et constituent les eaux les plus minéralisées de la zone d'étude. Il s'agit des eaux de Birnin Kazoe, Soubdou et Male-Male. Ces eaux sont caractérisées par des teneurs en dureté très faible, ce qui les classe parmi les eaux très douces.

La classe 2 renferme les eaux faiblement minéralisées, et représente $70 \%$ des eaux de la zone. Les eaux du puits de Kaotchouloum sont en plus caractérisées par des valeurs de turbidité, de fer dépassant les normes de l'OMS et des bactéries de types coliformes fécaux (Escherichia coli) et streptocoques fécaux.

\section{Résultats de la Classification Hiérarchique Ascendante (CHA)}

Les résultats de la Classification Hiérarchique Ascendante sont présentés par la Figure 4. Le dendrogramme obtenu montre trois classes statistiques distinctes. $\mathrm{La}$ Première classe est déterminée par: $\mathrm{Cl}^{-}$, dureté, $\mathrm{NO}_{3}{ }^{-}, \mathrm{HCO}_{3}{ }^{-}, \mathrm{CE}, \mathrm{Na}^{+}, \mathrm{F}^{-}, \mathrm{SO}_{4}{ }^{2-}, \mathrm{pH}$. Cette classe traduit le phénomène d'altération par hydrolyse des silicates. Les nitrates ont une origine superficielle et témoignent d'une pollution anthropique. La deuxième classe est déterminée par la turbidité, la couleur et les ions $\mathrm{Fe}^{2+}$ qui traduisent le phénomène d'oxydo-réduction. La troisième classe présente les ions $\mathrm{NO}_{2}^{-}$qui indiquent une contribution des activités anthropiques dans la minéralisation des eaux souterraines de la région.

L'analyse du dendrogramme permet de mettre en évidence deux phénomènes qui sont à la base du mécanisme d'acquisition du chimisme des eaux souterraines de la zone étudiée. Le phénomène majeur de la minéralisation dans la zone est l'altération par hydrolyse des silicates. Le deuxième phénomène est le phénomène d'oxydoréduction qui entraîne la précipitation du fer dans les eaux. 


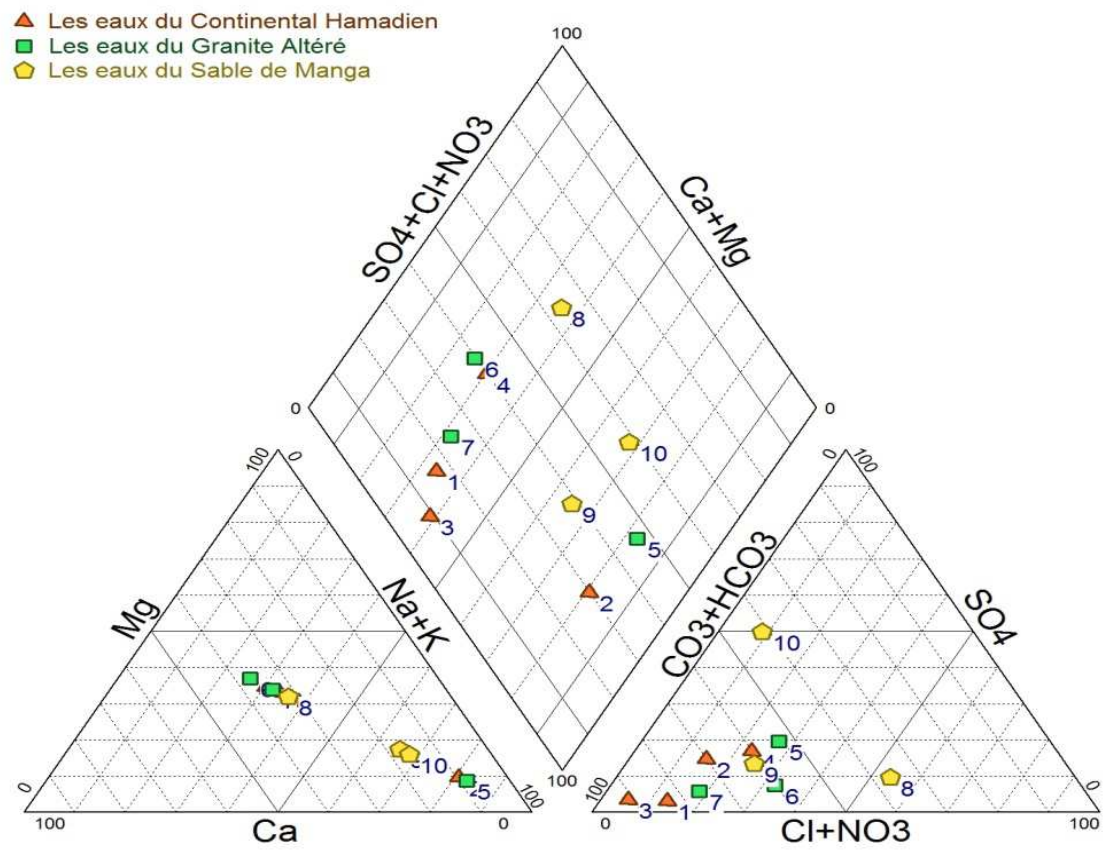

Figure 2: Classification des eaux souterraines à partir du diagramme de Piper.

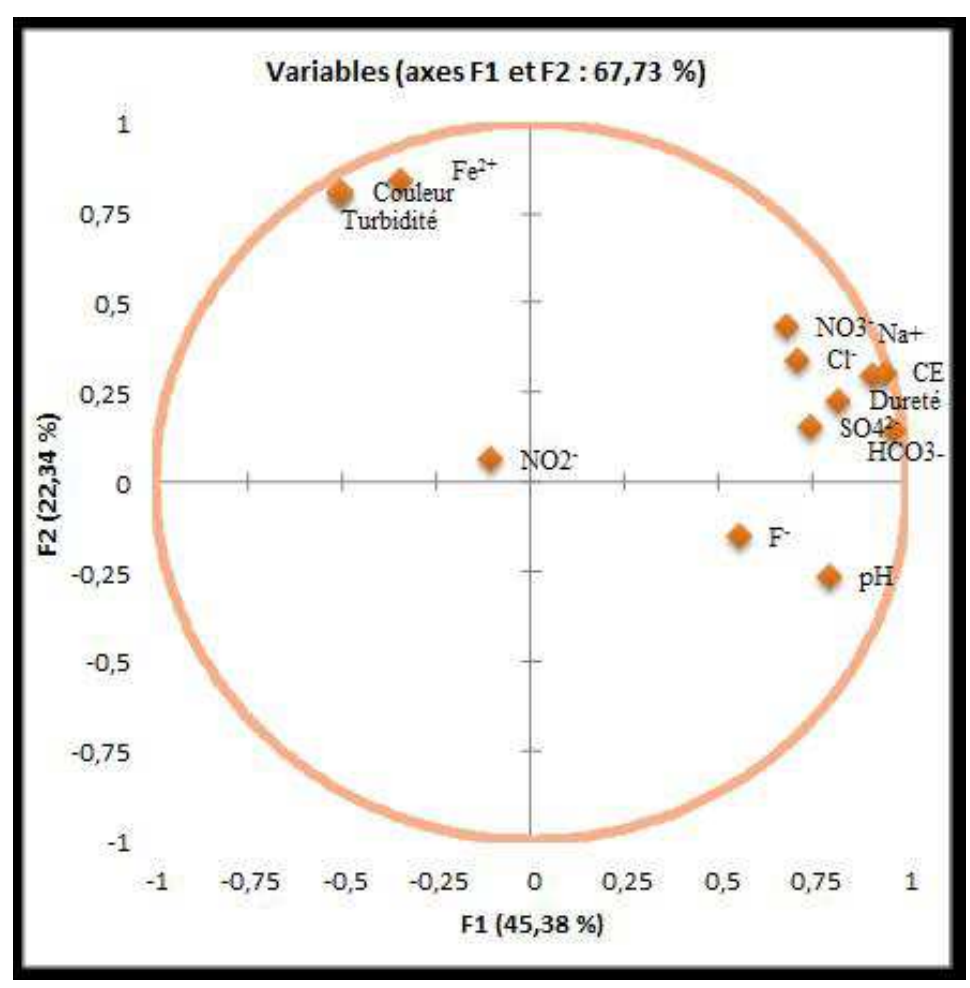

a) Analyse dans l'espace des variables (plan factoriel F1 - F2).

1910 
H. AMADOU et al. / Int. J. Biol. Chem. Sci. 8(4): 1904-1916, 2014

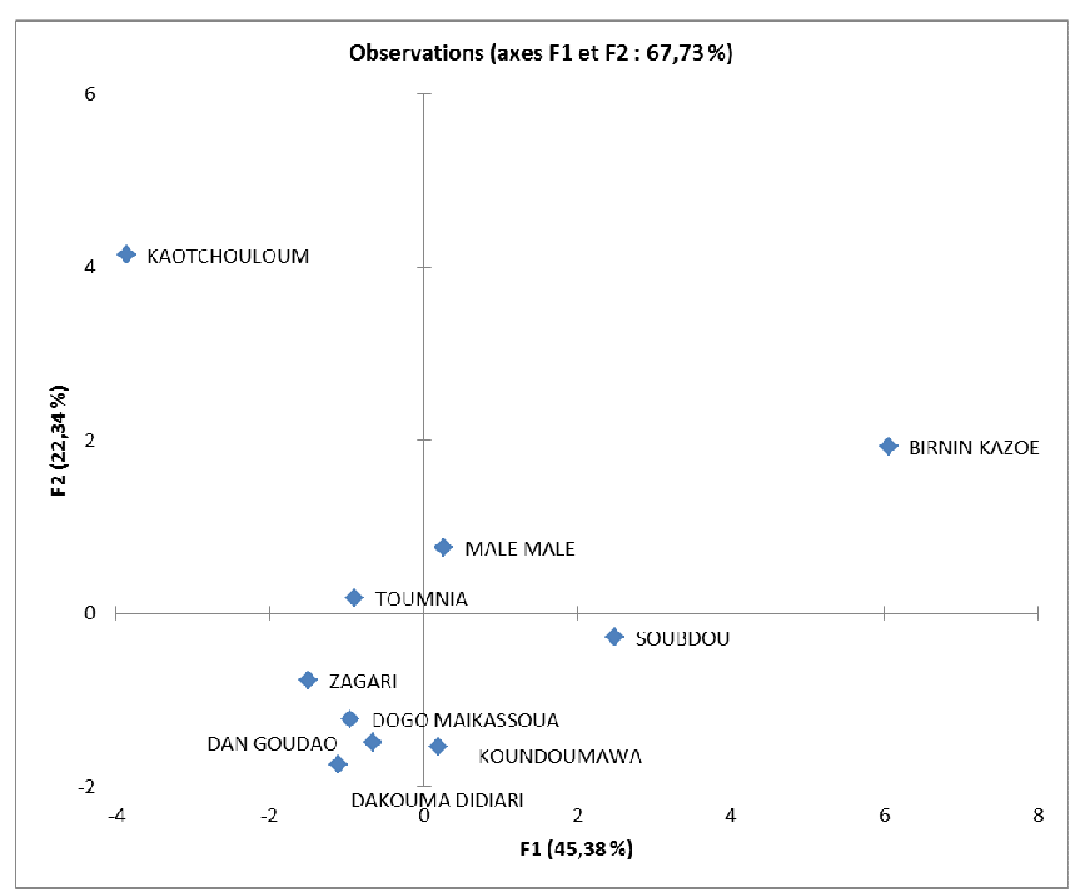

b) Analyse dans l'espace des unités statistiques (plan factoriel F1 - F2).

Figure 3 : Analyse en composantes principales des eaux dans le plan factoriel F1- F2.

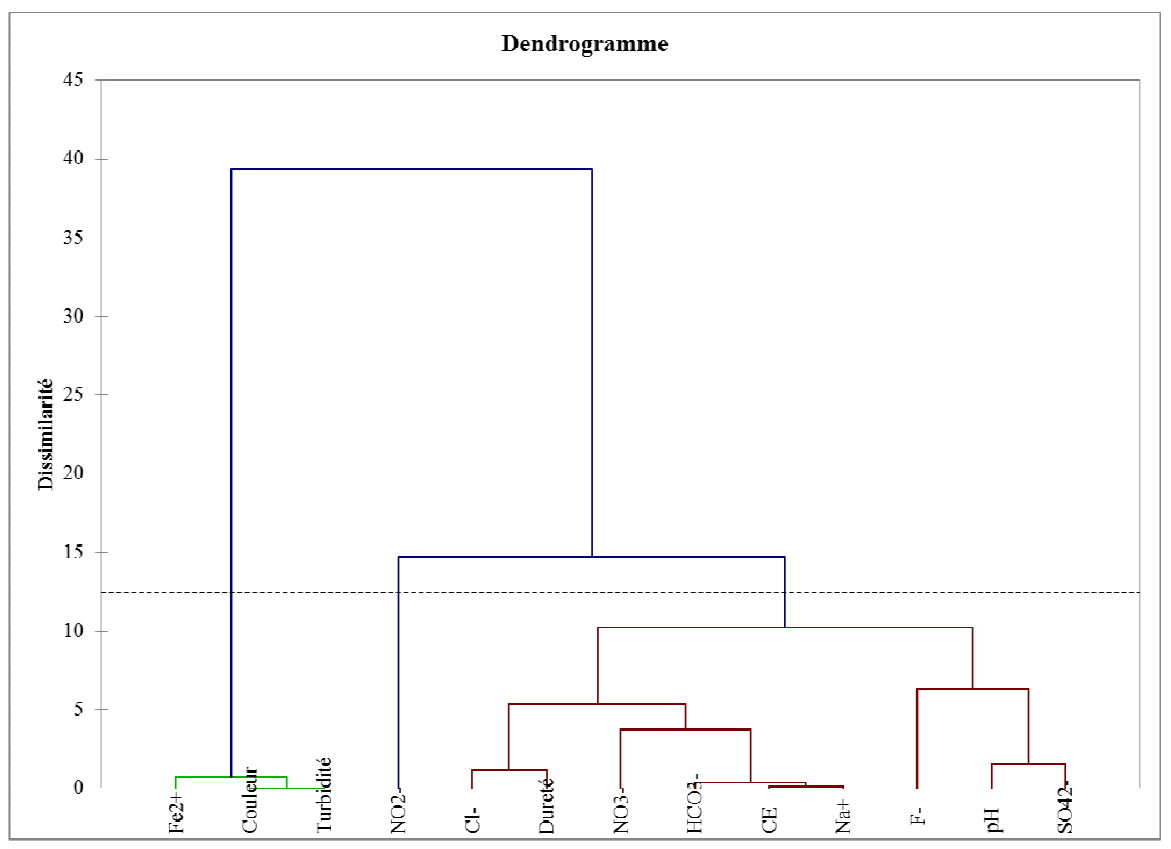

Figure 4 : Dendrogramme des eaux de la zone étudiée. 
Tableau 1 : Données physico-chimiques en mg/l des eaux souterraines de la région de Zinder.

\begin{tabular}{lcccccccc}
\hline Localité & $\mathbf{C . E}$ & $\mathbf{p H}$ & $\mathbf{T}{ }^{\circ} \mathbf{C}$ & $\begin{array}{c}\mathbf{C o u l e u r} \\
\mathbf{m g / l ~ P t}\end{array}$ & $\begin{array}{c}\text { Turb } \\
\text { NTU }\end{array}$ & $\mathbf{S O}_{\mathbf{4}}$ & $\mathbf{N O}_{\mathbf{2}}$ & $\mathbf{F e}^{\mathbf{2 +}^{+}}$ \\
\hline Dan Goudao & 223 & 6,86 & 30,4 & 0 & 0 & 4,08 & 0,00 & 0,03 \\
Koundoumawa & 335 & 7,15 & 29,6 & 0 & 0 & 23,67 & 0,00 & 0,06 \\
Dakouma Didiari & 195 & 6,85 & 30,5 & 0 & 0 & 3,60 & 0,00 & 0,03 \\
Kaotchouloum & 89 & 6,5 & 28,6 & 100 & 15 & 9,33 & 0,03 & 2,14 \\
Birnin Kazoe & 1955 & 7,2 & 29,5 & 0 & 0 & 127,35 & 0,04 & 0,46 \\
Toumnia & 249 & 6,76 & 28,2 & 0 & 0 & 7,42 & 1,04 & 0,03 \\
Dogo Maikassoua & 137 & 6,85 & 29,7 & 0 & 0 & 4,79 & 0,01 & 0,04 \\
Male Male & 517 & 6,8 & 28,7 & 20 & 2,5 & 23,43 & 0,01 & 0,09 \\
Zagari & 215 & 6,6 & 29,5 & 0 & 0 & 13,63 & 0,04 & 0,43 \\
Soubdou & 724 & 7,48 & 29,8 & 0 & 0 & 211,92 & 0,01 & 0,31 \\
\hline
\end{tabular}

Tableau 1 (suite): Données physico-chimiques en mg/l et bactériologiques (UFC/100ml) des eaux souterraines de la région de Zinder.

\begin{tabular}{lcccccccc}
\hline Localité & $\mathbf{F}$ & $\mathbf{N O}_{\mathbf{3}}$ & $\mathbf{H C O}_{\mathbf{3}}$ & $\mathbf{C l}$ & $\begin{array}{c}\text { Dureté } \\
{ }^{\circ} \mathbf{F}\end{array}$ & $\mathbf{N a}$ & Colif & Strep \\
\hline Dan Goudao & 0,23 & 8,19 & 136,64 & 8 & 7,30 & 17 & 0 & 0 \\
Koundoumawa & 1,70 & 0,00 & 142,74 & 18 & 3,20 & 62 & 0 & 0 \\
Dakouma Didiari & 0,08 & 0,55 & 122,00 & 4 & 5,50 & 16 & 0 & 0 \\
Kaotchouloum & 0,08 & 11,42 & 42,70 & 3 & 2,50 & 5 & 1800 & 230 \\
Birnin Kazoe & 1,13 & 40,74 & 439,20 & 107 & 18,00 & 395 & 38 & 0 \\
Toumnia & 0,20 & 27,71 & 76,86 & 8 & 8,10 & 13 & 154 & 12 \\
Dogo Maikassoua & 0,50 & 12,56 & 79,30 & 4 & 5,60 & 12 & 12 & 0 \\
Male Male & 0,31 & 5,24 & 112,24 & 94 & 15,50 & 40 & 17 & 0 \\
Zagari & 0,32 & 0,13 & 79,30 & 19 & 4,10 & 35 & 12 & 0 \\
Soubdou & 0,50 & 23,61 & 225,70 & 14 & 11,00 & 106 & 10 & 0 \\
\hline
\end{tabular}

\section{DISCUSSION}

L'étude des paramètres physicochimiques a montré la grande variabilité chimique des eaux souterraines. Cette étude a révélé également que les eaux souterraines sont physico chimiquement conformes dans l'ensemble aux normes recommandées par l'OMS (OMS, 2008) et ne présentent pas de danger majeur pour la consommation humaine. Cependant, il existe de fortes teneurs en fer dans les eaux de certaines localités (40\% des points d'eaux analysés). Le fer peut être en solution vraie à l'état colloïdal, plus ou moins combiné à la matière organique, sous forme de complexes organiques ou minéraux ou sous forme de particules en suspension. Au contact de l'air, l'ion ferreux $\mathrm{Fe}^{2+}$ (soluble) est oxydé en ion ferrique $\mathrm{Fe}^{3+}$ (insoluble). Ainsi, la présence de l'oxyde de fer contenu dans l'eau confère à l'eau sa turbidité et sa couleur rouge (Lasm et al., 2008).

L'étude hydro-chimique a permis de mettre en évidence la prédominance de faciès bicarbonaté dans les eaux souterraines de la zone de Zinder. Ce résultat est en accord avec celui de plusieurs auteurs ayant travaillé en Afrique de l'Ouest et qui ont tous abouti à la conclusion selon laquelle, le faciès bicarbonaté est le plus représenté dans les eaux du socle (Soro, 2002; Ahoussi, 2008; Oga et al., 2009; Chemseddine et al., 2009; Kouassi et al., 2010 ; Yao et al., 2010; Lasm et al., 2011). 
Tableau 2: Matrice de corrélation.

\begin{tabular}{|c|c|c|c|c|c|c|c|c|c|c|c|c|c|}
\hline Variables & CE & pH & Couleur & Turb & $\mathrm{SO}_{4}{ }^{2-}$ & $\mathrm{NO}_{2}^{-}$ & $\mathrm{Fe}_{2}^{+}$ & $\mathbf{F}^{-}$ & $\mathrm{NO}_{3}^{-}$ & $\mathrm{HCO}_{3}^{-}$ & $\mathrm{Cl}^{-}$ & Dureté & $\mathrm{Na}^{+}$ \\
\hline $\mathrm{CE}$ & 1 & & & & & & & & & & & & \\
\hline $\mathrm{pH}$ & 0,581 & 1 & & & & & & & & & & & \\
\hline Couleur & $-0,230$ & $-0,511$ & 1 & & & & & & & & & & \\
\hline Turb & $-0,232$ & $-0,508$ & 0,999 & 1 & & & & & & & & & \\
\hline $\mathrm{SO}_{4}{ }^{2-}$ & 0,658 & 0,827 & $-0,188$ & $-0,186$ & 1 & & & & & & & & \\
\hline $\mathrm{NO}_{2}^{-}$ & $-0,113$ & $-0,185$ & $-0,117$ & $-0,114$ & $-0,16$ & 1 & & & & & & & \\
\hline $\mathrm{Fe}_{2}^{+}$ & $-0,055$ & $-0,402$ & $\mathbf{0 , 9 3 7}$ & 0,944 & $-0,02$ & $-0,156$ & 1 & & & & & & \\
\hline $\mathrm{F}^{-}$ & 0,469 & 0,587 & $-0,315$ & $-0,311$ & 0,278 & $-0,208$ & $-0,225$ & 1 & & & & & \\
\hline $\mathrm{NO}_{3}^{-}$ & 0,741 & 0,424 & $-0,081$ & $-0,075$ & 0,597 & 0,397 & 0,067 & 0,133 & 1 & & & & \\
\hline $\mathrm{HCO}_{3}^{-}$ & 0,968 & 0,702 & $-0,337$ & $-0,334$ & 0,700 & $-0,196$ & $-0,146$ & 0,515 & 0,686 & 1 & & & \\
\hline $\mathrm{Cl}^{-}$ & 0,791 & 0,246 & $-0,106$ & $-0,126$ & 0,313 & $-0,161$ & $-0,099$ & 0,325 & 0,405 & 0,668 & 1 & & \\
\hline Dureté & 0,813 & 0,464 & $-0,276$ & $-0,293$ & 0,549 & 0,012 & $-0,262$ & 0,136 & 0,638 & $\mathbf{0 , 7 3 7}$ & 0,872 & 1 & \\
\hline $\mathrm{Na}^{+}$ & 0,987 & 0,550 & $-0,212$ & $-0,209$ & 0,616 & $-0,146$ & $-0,006$ & 0,523 & 0,717 & 0,966 & 0,729 & 0,714 & 1 \\
\hline
\end{tabular}

Tableau 3: Valeurs propres et pourcentages exprimés pour les axes principaux.

\begin{tabular}{lll}
\hline & F1 & F2 \\
\hline Valeur propre & 6,35 & 3,12 \\
\% Total de la variance exprimée & 45,38 & 22,34 \\
Cumul de la variance exprimée $(\%)$ & 45,38 & 67,72 \\
\hline
\end{tabular}


La dominance du faciès bicarbonaté dans les eaux de socle est à rattacher à l'origine de la production des ions bicarbonatés. L'abondance des ions bicarbonates dans ces eaux peut s'expliquer par l'hydrolyse des feldspaths.

L'analyse statistique multi variée des données hydro chimiques (ACPN et CHA), a permis d'identifier deux principaux mécanismes qui sont responsables de l'évolution de la minéralisation des eaux de la zone. Le mécanisme à la base de la minéralisation en éléments métalliques des eaux est le phénomène d'oxydo-réduction. Le deuxième mécanisme est la minéralisation naturelle. Selon Soro (2010), la minéralisation naturelle reste le processus majeur qui gouverne la chimie des eaux souterraines. Les éléments qui définissent ce facteur proviennent d'une longue durée de mise en solution suite au contact eau-roche. Ces variables sont issues de l'altération des roches et de l'hydrolyse des minéraux (Savané et Soro, 2001). Nous pouvons ainsi caractériser les trois aquifères échantillonnés:

Le Continental Hamadien présente des eaux appartenant à la classe 2, faiblement minéralisées. Ces eaux ont été échantillonnées dans les villages de Dan-Goudao, Koundoumawa, Dakouma-Didiari et Kaotchouloum. On note une forte teneur en fluor à Koundoumawa et une forte teneur en fer à Kaotchouloum avec une présence des bactéries de types coliformes et streptocoques fécaux.

Le Granite Altéré : les eaux ont été échantillonnées dans les villages de BirninKazoé, Toumnia, et Dogo-Maikassoua et sont des eaux variables. Le premier village (BirninKazoé) présente une eau de classe 1 fortement minéralisée avec une concentration en fer dépassant les normes de l'OMS, des taux de nitrate et de fluor proches de la limite fixée par l'OMS. Les eaux des deux autres villages (Toumnia, et Dogo-Maikassoua) appartiennent à la classe 2, faiblement minéralisées.

Les Sables du Manga: les eaux de ces aquifères ont des caractéristiques variables d'un point à un autre. Zagari présente une eau faiblement minéralisée douce mais ferrugineuse (Concentration en fer de 0,43 $\mathrm{mg} / \mathrm{l})$. Le village de Soubdou présente une eau très minéralisée, très carbonatée, et dont les concentrations en sodium et sulfate sont voisines de la limite fixée par l'OMS. MaléMalé présente une eau appartenant à la classe 1 donc minéralisée et à la limite moyennement dure.

\section{Conclusion}

L'étude des paramètres physicochimiques a montré la grande variabilité chimique des eaux souterraines. Les eaux étudiées sont conformes dans l'ensemble aux normes recommandées par l'OMS et ne présentent pas de danger majeur pour la consommation humaine. Cependant, il existe de fortes teneurs en fer dans les eaux de certaines localités. Ces fortes teneurs peuvent être atténuées par le traitement au charbon actif, ce qui permettra de supprimer la coloration des eaux. La classification des résultats d'analyses chimiques obtenues à partir du diagramme triangulaire de Piper permet d'identifier quatre groupes d'eau. Les eaux bicarbonatées calciques et magnésiennes qui sont les plus importantes $(50 \%)$ des eaux étudiées, les eaux bicarbonatées sodipotassiques $(30 \%)$, les eaux chlorurées calciques et magnésiennes $(10 \%)$ et les eaux sulfatées sodi-potassiques $(10 \%)$. Les bicarbonates se révèlent comme les ions prédominants. L'Analyse en Composantes Principales et la Classification Hiérarchique Ascendante (CHA) indiquent que la minéralisation des eaux étudiées est contrôlée par deux grands phénomènes: la minéralisation temps de séjour ou l'hydrolyse pour les eaux souterraines, et le phénomène 
d'oxydo-réduction. Par ailleurs, les eaux de la zone d'étude contiennent de forts taux de coliformes fécaux (Escherichia coli) et de streptocoques fécaux qui sont la conséquence d'une pollution d'origine humaine récente.

\section{RÉFÉRENCES}

Ahoussi KE, Koffi YB, Kouassi AM, Soro G, Biemi J. 2013. Étude hydro chimique et microbiologique des eaux de source de l'ouest montagneux de la Côte d'Ivoire : Cas du village de Mangouin-Yrongouin (Sous-préfecture de Biankouman). Journal of Applied Biosciences, 63: 4703-4719.

Ahoussi KE, Oga YMS, Koffi YB, Kouassi AM, Soro N, Biemi J. 2011. Caractérisation hydrogéochimique et microbiologique des ressources en eau du site d'un Centre d'Enfouissement Technique (CET) de Côte d'Ivoire : cas du CET de Kossihouen dans le District d'Abidjan (Côte d'Ivoire). International Journal of Biological and Chemical Sciences, 5(5): 2114-2132.

Ahoussi KE. 2008. Evaluation quantitative et qualitative des ressources en eau dans le Sud de la Côte d'Ivoire. Application de l'hydrochimie et des isotopes de l'environnement à l'étude des aquifères continus et discontinus de la région d'Abidjan-Agboville. Thèse de Doctorat de l'Université de Cocody-Abidjan, Côte d'Ivoire, p.270.

Ahoussi MA, Koffi EK, Ake BY, Biemi J. 2012. Caractérisation hydrogéo-chimique des eaux des aquifères fissurés de la zone Guiglo-Duekoué (Ouest de la Côte d'Ivoire). International Journal of Biological and Chemical Sciences, 6(1): 504-518.

Biémi J. 1992. Contribution à l'étude géologique, hydrogéologique et par télédétection des bassins versants Subsahéliens du socle précambrien d'Afrique de de l'Ouest : Hydro structurale, hydrochimie et isotopie des aquifères discontinus des sillons et aires granitiques de la Haute Marahoué (Côte d'Ivoire). Thèse de Doctorat d'Etat ès Sciences Naturelle, Université Nationale de Côte d'Ivoire, p. 480.

Chemseddine F, Abderrahmane B, Abdelkader R, Elias S. 2009. Caractérisation hydrogéochimique des eaux souterraines du complexe aquifère Morsott-Laouinet (Région Nord de Tébessa, Sud-Est algérien). Afrique Science, 05(2): 217 - 231.

Eblin SG, Sombo AP, Soro G, Aka N, Kambiré O, Soro N. (2014) Hydrochimie des eaux de surface de la région d'Adiaké (sud-est côtier de la Côte d'Ivoire). Journal of Applied Biosciences, 75: 62596271.

Greiger J, Pougnet R. 1967. Notice explicative sur géologique de la republique du Niger à l'échelle 1:2 000 000. Editions du bureau de recherches géologiques et miniéres, 62 p.

Jain P, Sharma JD, Sohu D, Sharma P. 2005. Chemical analysis of drinking water of villages of Sanganer Tehsil, Jaipur District. Int. J. Environ. Sci. Tech., 2(4): 373-379.

Kortatsi BK. 2007. Hydrochemical framework of groundwater in the Ankobra Basin, Ghana. Aquatic Geochemistry, 13(1): 4174.

Kouassi AM, Yao KA, Ahoussi KE, Seki CL, Yao NA, Kouassi KI. 2010. Apports des méthodes statistiques et hydrochimiques à la caractérisation des eaux des aquifères fissurés de la région du N'zi-Comoé (Centre-Est de la Côte d'Ivoire). International Journal of Biological and Chemical Sciences, 4(5): 1816-1838.

Lasm T, ASM, Lasme O, Oga MS, Youanta M, Baka D, Kouamé KF, Yao KT. 2011. Caractérisation hydrochimique des aquifères fissurés de la région de San- 
Pedro (Sud-Ouest de la Côte d'Ivoire). International Journal of Biological and Chemical Sciences, 5(2): 642-662.

Mor S, Ravindra K, Dahiya RP, Chandra A. 2006. Leachate characterization and assessment of groundwater pollution near municipal solid waste landfill site. Environ Monit Assess, 118: 435-456.

O.M.S. 2008. Guidelines for Drinking-Water Quality ( $3^{\text {rd }}$ edn). OMS: Geneva; 515.

Oga MS, Lasm T, Yao KT, Soro N, Saley MB, Kouassi D, Gnamba F. 2009. Caractérisation chimique des eaux des aquifères de Fracture: cas de la région de Tiassalé en Côte D'ivoire. European Journal of Scientific Research, 31(1): 7287.

Ousmane B. 1978. Contribution à l'étude hydrogéologique des regions de socle du sahel: l'hydrogéologie du Damagaram occidental (environs de Zinder-Niger). Thèse de doctorat de $3^{\mathrm{e}}$ cycle, Université de Montpellier, $123 \mathrm{p}$.

Savané I, Soro N. 2001. Caractérisation chimique et isotopique des eaux souterraines du Nord-Ouest de la Côte d'Ivoire: recharge des aquifères discontinus de la région d'Odienné. Africa Géoscience Review, 8(4): 379-390.

Soro N. 2002. Hydrochimie et géochimie isotopique des eaux souterraines du degré carré de Grand-Lahou et ses environs (sudouest de la Côte d'Ivoire). Implication hydrologique et hydrogéologique. Thèse d'Etat, Université de Cocody, p. 272.

Soro. G. 2010. Évaluation quantitative et qualitative des ressources en eaux souterraines dans la région des lacs (centre de la côte d'ivoire) : Hydrogéologie et hydrochimie des aquifères discontinus du district de Yamoussoukro et du département de Tiebissou. Thèse de Doctorat de l'Université de CocodyAbidjan, Côte d'Ivoire, 257p.

Yao KA, Kouassi AM, Koffi YB, Biemi J. 2010. Caractérisation hydrodynamique et hydrogéochimique des aquifères fissurés de la région de Toumodi (Centre de la Côte d'Ivoire). Journal of Environmental Hydrology, 18(26): 1-15. 